\title{
A thermodynamic model of nanofluid viscosity based on a generalized Maxwell-type constitutive equation
}

G. Lebon and H. Machrafi

Liège University, Thermodynamics of Irreversible Processes, Allée du 6-Août, 19, 4000 Liège, Belgium

\begin{abstract}
An original study of nanofluid viscosity is proposed. The carrier fluid is assumed Newtonian but the two-phase nanofluid displays properties typical of a generalized Maxwell constitutive law. Our approach is based on an extension of Einstein's model describing suspensions of solid particles in fluids by the introduction of the following elements: presence of a layer around the nanoparticles and a thermodynamic description of the role of size effects. The theoretical formalism is applied to liquid argon with lithium nanoparticles and to alumina nanoparticles in water. Good agreement with experimental data and molecular dynamics simulation is observed.
\end{abstract}

Keywords: nanofluids, viscosity, generalized Maxwell model, boundary layer, particle's size, extended thermodynamics 


\section{Introduction.}

Nanofluids are binary mixtures consisting of nanoparticles dispersed in a host fluid. These systems have met an increasing interest in several industrial applications, like in biotechnology, nanotechnology, electromechanical systems, they have proved to be relevant in the developments of new drugs, paints, lubricants among others. It is well know that the presence of nanoparticles influences considerably the thermo-mechanical properties of the basic fluid like, in particular, thermal conductivity and viscosity.

In this paper, focus is put on the role of nanoparticles on the shear viscosity of the system, an impressive lot of works (e.g. [1-10]) have been published on the subject. Viscosity depends essentially on the temperature, the nature of the particles and the fluid, the volume fraction of particles and their size. Subsequently, the analysis is focused on the role of volume fraction and particles size, the temperature is assumed uniform.

It has been observed that the viscosity of nanofluids is much lager that that of the host fluid. Several theoretical and ad hoc expressions for the viscosity $\eta$ in terms of the particle's volume fractions $\varphi$ have been proposed, among which the celebrated Einstein formula [11]

$$
\eta=\eta_{f}(1+2.5 \varphi)
$$

with $\eta_{f}$ denoting the viscosity of the host fluid, this expression is valid for dilute mixtures

$(\varphi<0.05)$ with spherical particles. Other more sophisticated ad-hoc relations have been formulated like a quadratic dependence in the viscosity [12]

$$
\eta=\eta_{f}\left(1+a_{1} \varphi+a_{2} \varphi^{2}\right)
$$


wherein $a_{1}$ and $a_{2}$ are ad hoc parameters taking different values according to the nature of the nanofluid. Other models like those of Chen et al [5] and Krieger and Dougherty [ 8 ] have also been exploited.

The dependence of viscosity on the size of nanoparticles has been a subject of debate. For some authors $[13,14]$, viscosity increases with increasing dimensions of nanoparticles while others [15-17] assert that viscosity diminishes with increasing size. For sufficiently large particles, the dependence with respect to size becomes negligible. It is however worth to mention that no valuable theoretical considerations, outside molecular dynamic simulations [18] are able to explain such behaviors.

The gap will be filled in the forthcoming: a formalism based on Extended Irreversible Thermodynamics (EIT) [19-21] is presented wherein the nanofluid is viewed as a generalized Maxwell model. In addition, our analysis will emphasize the role of interfacial layers surrounding the solid particle, acting as a possible mechanism for momentum transfer. This concept was introduced by Choi [22] and exploited by Yu and Choi [23] and Xie et al [24] to interpret the enhancement of thermal conductivity in nanofluids.

The working hypotheses of our model are the following:

- the dispersed particles are solid spheres of radius $r$,

- the host fluid is homogeneous, isotropic and Newtonian,

- the particles are homogeneously distributed in the fluid,

- no formation of aggregates,

- the effects of the Brownian motion of nanoparticles is neglected,

- thermal effects are not taken into account and temperature is uniform. 
The paper is organized as follows. In Section 2, the main ingredients underlying EIT are recalled and the evolution equations governing the behavior of the state equations, essentially the internal energy and the viscous pressure tensor, are formulated. In Section 3, an expression of the effective shear viscosity of nanofluids in presence of spherical rigid nanoparticles is derived. In Section 4, the model is applied to lithium particles dispersed in Argon and to aluminate particles in water respectively. Conclusions are drawn in Section 5.

\section{Extended Irreversible Thermodynamics}

The description of systems at subscales, such as nanoparticles, requires to go beyond the classical theory of irreversible processes [25]. A good candidate for treating these classes of problems is Extended Irreversible Thermodynamics (EIT). The principal idea behind EIT is to elevate the fluxes, as the fluxes of mass, energy and momentum to the status of independent variables at the same level as the classical conserved variables like mass, energy or momentum.

As a case-study, let us consider the flow of a viscous incompressible fluid at uniform temperature. The generalization to more complicated systems as fluids mixtures [26], polymer solutions [27], suspensions [28], porous media [29] and others have been dealt with in detail in numerous publications and books. In the case of an incompressible fluid flow, the only relevant conserved variable is the specific internal energy $e$ (per unit mass)) whereas the corresponding flux variable is the viscous pressure tensor $\boldsymbol{P}$, it is a second order symmetric traceless tensor, in contrast with $e$, it is not a conserved quantity. The corner stone of EIT is to assume the existence of a specific non-equilibrium entropy function $s$ depending on both $e$ and $\boldsymbol{P}$ so that $s=s(e, \boldsymbol{P})$ or, in terms of time derivatives, 


$$
d_{t} s=\frac{\partial s}{\partial e} d_{t} e+\frac{\partial s}{\partial \boldsymbol{P}} \otimes d_{t} \boldsymbol{P}
$$

wherein $\otimes$ stands for the inner product of the corresponding tensors, the symbol $d_{t}$ designates the time derivative which is indifferently the material or the partial time derivative as the system is, respectively, in motion or at rest. It is assumed that $s$ is a concave function of the variables and that it obeys a general time-evolution equation which can be written in the form

$$
\sigma^{s}=\rho d_{t} s+\nabla . J^{s} \geq 0
$$

with $\sigma^{s}$ its rate of production per unit volume (in short, the entropy production) to be positive definite in order to satisfy the second principle of thermodynamics, $\rho$ is the mass density of the nanofluid and the vector $\boldsymbol{J}^{\boldsymbol{s}}$ is the entropy flux, the dot between $\nabla$ and $\boldsymbol{J}^{\boldsymbol{s}}$ denotes the scalar product. Let us define the temperature by $T^{-1}=\partial s / \partial e$ assumed to be independent of the dissipative flux $\boldsymbol{P}$, next, we select the constitutive equation for $\partial \eta / \partial \boldsymbol{P}$ as assumed to be given by the linear relation $\partial s / \partial \boldsymbol{P}=-\left(\gamma_{1} / \rho\right) \boldsymbol{P}$, where $\gamma_{1}$ is a material coefficient depending generally on $\rho$ and $T, \gamma$ is positive definite in order to meet the property that $s$ is maximum at local equilibrium, the minus sign in front of $\gamma(T) \boldsymbol{P}$ has been introduced for convenience. Under these conditions, expression (3), can be written as

$$
\rho d_{t} s=-T^{-1} \boldsymbol{P} \otimes \boldsymbol{D}-\gamma_{1} \boldsymbol{P} \otimes d_{t} \boldsymbol{P}
$$

after use has been made of the energy conservation law

$$
\rho d_{t} e=-\boldsymbol{P} \otimes \boldsymbol{D}
$$


wherein $\boldsymbol{D}$ is the symmetric traceless velocity gradient tensor. At the actual order of approximation and in absence of heat flux, the entropy flux is zero and the entropy production takes the form

$$
\sigma^{s}=-\boldsymbol{P} \otimes\left(T^{-1} \boldsymbol{D}+\gamma_{1} d_{t} \boldsymbol{P}\right) \geq 0
$$

It is a bilinear relation in the flux $\boldsymbol{P}$ and the quantity represented by the two terms between the parentheses that is usually called the thermodynamic force $\boldsymbol{X}$. The simplest way to guarantee the positiveness of the entropy production $\sigma^{s}$ is to assume a linear flux-force relation of the form $\boldsymbol{X}=-\mu_{1} \boldsymbol{P}$ with $\mu_{1}$ a positive phenomenological coefficient, this procedure leads to the wellknown Maxwell model

$$
\tau_{1} d_{t} \boldsymbol{P}=-\boldsymbol{P}-2 \eta \boldsymbol{D},
$$

after one has put $\gamma_{1} / \mu_{1}=\tau_{1}$ (relaxation time) and $1 / T \mu_{1}=2 \eta$ (shear viscosity) and wherein $\tau_{1}$ and $\eta$ are positive quantities as $\mu_{1}$ and $\gamma_{1}$ have been proven to be positive coefficients. Letting $\tau_{1}$ vanish, one finds back Newton's law $\boldsymbol{P}=-2 \eta \boldsymbol{D}$. Although Maxwell's relation is useful at short time scales (high frequencies), it is not satisfactory with the purpose to describe short length scales wherein non-localities play a preponderant role, for instance fluids in presence of nanoparticles.

In more complex materials like in nanofluids, fluxes of higher order should be introduced as extra states variables. Non-local effects, which are important in presence of nanoparticles, are elegantly accounted for, in the framework of EIT, by appealing to a hierarchy of fluxes $\boldsymbol{P}^{(2)}(\equiv \boldsymbol{P}), \boldsymbol{P}^{(3)}, \ldots \boldsymbol{P}^{\mathrm{N})}$ where the second order tensor $\boldsymbol{P}^{(2)}$ is identified with the usual viscous pressure tensor $\boldsymbol{P}, \boldsymbol{P}^{(3)}$ (a tensor of rank three) is the flux of the pressure tensor, ...etc. 
Here for simplicity, we limit our developments to the use of $\boldsymbol{P}$ and $\boldsymbol{P}^{(3)}$ as flux state variables but there will be no difficulty to include higher order tensors as $\boldsymbol{P}^{(4)}, \ldots \quad \boldsymbol{P}^{(\mathrm{N})}$ as done previously in the problem of non-local heat conduction wherein an infinite number of extra fluxes have been introduced [30-32]. From the kinetic theory point of view, $\boldsymbol{P}^{(2)}$ and $\boldsymbol{P}^{(3)}$ represent the second and third order moments of the velocity distribution. Written in Cartesian coordinates and designating by $f$ the distribution function, the fluxes $\boldsymbol{P}^{(2)}$ and $\boldsymbol{P}^{(3)}$ read as

$$
P_{i j}^{(2)} \equiv P_{i j}=\int C_{i} C_{j} f d c, \quad P_{i j k}^{(3)}=\int C_{i} C_{j} C_{k} f d c
$$

with $\boldsymbol{C}=\boldsymbol{c}-\boldsymbol{v}$ the relative velocity of particles with respect to their mean velocity $\boldsymbol{v}$.

Up to the third-order moment approximation, which is sufficient for the present purpose, the Gibbs equation generalizing expression (3) takes the form

$$
d_{t} s\left(e, \boldsymbol{P}, \boldsymbol{P}^{(3)}, \ldots\right)=T^{-1} d_{t} e-\left(\gamma_{1} / \rho\right) \boldsymbol{P} \otimes d_{t} \boldsymbol{P}-\left(\gamma_{2} / \rho\right) \boldsymbol{P}^{(3)} \otimes d_{t} \boldsymbol{P}^{(3)}
$$

while the entropy flux is no longer equal to zero but is given by

$$
\boldsymbol{J}^{s}=\beta \boldsymbol{P}^{(3)} \otimes \boldsymbol{P}
$$

with $\beta$, a phenomenological coefficient allowed to depend on $e$ and the volume fraction of the paticles but not on the flux variables. The entropy production (4) is obtained by substitution of $d_{t} s$ and $\boldsymbol{J}^{s}$ from (10) and (11) respectively and elimination of $d_{t} e$ via the energy balance (4), the result is

$$
\sigma^{s}=-\boldsymbol{P} \otimes\left(T^{-1} \boldsymbol{D}+\gamma_{1} d_{t} \boldsymbol{P}-\beta \nabla \cdot \boldsymbol{P}^{(3)}\right)-\boldsymbol{P}^{(3)} \otimes\left(\gamma_{2} d_{t} \boldsymbol{P}^{(3)}-\beta \nabla \boldsymbol{P}\right) \geq 0
$$


The above bilinear expression in fluxes and forces (the quantities between parentheses) suggests the following linear flux-force equations

$$
\begin{gathered}
\gamma_{1} d_{t} \boldsymbol{P}+\mu_{1} \boldsymbol{P}=-T^{-1} \boldsymbol{D}+\beta \nabla \cdot \boldsymbol{P}^{(3)}, \\
\gamma_{2} d_{t} \boldsymbol{P}^{(3)}+\mu_{2} \boldsymbol{P}^{(3)}=\beta \nabla \boldsymbol{P},
\end{gathered}
$$

wherein $\gamma_{n}, \beta$ and $\mu_{n}(n=1,2)$ are phenomenological coefficients allowed to depend in particular on the temperature and/or the relative volume fraction of the constituents. Relations (13) and (14) can also be viewed as time evolution equations for the fluxes $\boldsymbol{P}$ and $\boldsymbol{P}^{(3)}$. Making use of (13) and (14), expression (12) of the entropy production reads as

$$
\sigma^{s}=\mu_{1} \boldsymbol{P} \otimes \boldsymbol{P}+\mu_{2} \boldsymbol{P}^{(3)} \otimes \boldsymbol{P}^{(3)} \geq 0,
$$

from which follows that $\mu_{1} \geq 0$ and $\mu_{2} \geq 0$ to satisfy the positiveness of the entropy production.. Identifying the quantities $\gamma_{1} / \mu_{1}=\tau_{1}$ and $\gamma_{2} / \mu_{2}=\tau_{2}$ with $\tau_{1}$ and $\tau_{2}$, the positive relaxation times of the pressure tensors $\boldsymbol{P}$ and $\boldsymbol{P}^{(3)}$ respectively and, assuming that $\tau_{2}<<\tau_{1}$, as confirmed by the kinetic theory of gases, equation (14) leads to

$$
\boldsymbol{P}^{(3)}=\frac{\beta}{\mu_{2}} \nabla \boldsymbol{P}
$$

Substitution of this result in (13) and making use of the identification $2 \eta=\left(\mu_{1} T\right)^{-1}$ yields

$$
\tau_{1} d_{t} \boldsymbol{P}+\boldsymbol{P}=-2 \eta \boldsymbol{D}+\frac{\beta^{2}}{\mu_{1} \mu_{2}} \nabla^{2} \boldsymbol{P}
$$

The last term accounts for the non-local effects related to the presence of nanoparticles. In a previous works [19,31,33], general relations between the various phenomenological 
coefficients were obtained. Through dimensional analysis, it was inferred that $\beta=-\tau_{1} \mu_{1}$ and $\mu_{2}=\left(\frac{\tau_{1}}{L}\right)^{2} \mu_{1}$, with $L$ denoting a characteristic reference length, related to the mean free path $l$ of the momentum carriers. Moreover, in a nanofluid (a two-component system), the reference length $L$ will generally be dependent on the volume fraction of the nanoparticles, i.e. $L=$ $L(\varphi, l)$. At the simplest first-order approximation in both $l$ and $\varphi$, one has $L=l \varphi$. In virtue of the above results, we may write

$$
\beta^{2} / \mu_{1} \mu_{2}(\geq 0)=l^{2} \varphi^{2}
$$

This coefficient fulfills the following three requirements: it is positive definite, it has the dimension of a length to the square, it vanishes in absence of nanoparticles, i.e for $\varphi=0$. Substituting expression (18) in (17), one obtains the time-evolution equation of the pressure tensor $\boldsymbol{P}$,

$$
\tau_{1} d_{t} \boldsymbol{P}+\boldsymbol{P}=-2 \eta \boldsymbol{D}+l^{2} \varphi^{2} \nabla^{2} \boldsymbol{P}
$$

Introduction of the factor $\varphi^{2}$ in (18) is justified, because, as recalled above, a-priori all the phenomenological coefficients are $\varphi$-dependent as we are faced with a two-component system formed by a fluid and nanoparticles; a dependence in $\varphi^{2}$ is not new and was also shown in the constitutive equation of the pressure tensor in polymer blends (e.g. [27,34]). It is the introduction in (19) of the factor $\varphi$ which accounts explicitly for the dependence of the constitutive equation on the nanoparticles; for $\varphi=0$ and at steady states expression (19) reduces to the classical Newton law $\boldsymbol{P}=-2 \eta \boldsymbol{D}$ for a Newtonian fluid. 
Relation (19) is the key one of our formalism and is the equivalent of the GuyerKrumhansl [35] equation used to describe nonlocal effects in heat transport wherein the flux variable $\boldsymbol{P}$ is replaced by the heat flux vector $\boldsymbol{q}[19-21]$.

In the next section, we will determine the dependence of the effective viscosity of the nanofluid with respect to the size of the nanoparticles.

\section{Effective viscosity of nanofluids}

After application of the spatial Fourier transforms $\hat{\boldsymbol{P}}(\boldsymbol{k}, t)=\int_{-\infty}^{+\infty} \boldsymbol{P}(\boldsymbol{r}, t) e^{-i \boldsymbol{k} \cdot \boldsymbol{r}} d \boldsymbol{r}$ and $\hat{\boldsymbol{v}}(\boldsymbol{k}, t)=\int_{-\infty}^{\infty} \boldsymbol{v}(\boldsymbol{r}, t) e^{-i \boldsymbol{k} . \boldsymbol{r}} d \boldsymbol{r}$ to relation (19), with $\boldsymbol{k}$ designating the wave-number vector, $\boldsymbol{r}$ the position vector and $\boldsymbol{v}(\boldsymbol{r}, t)$ the vectorial velocity field, one is led to the following time-evolution equation of the Fourier transformed fluxes

$$
\tau_{1} d_{t} \hat{\boldsymbol{P}}+\hat{\boldsymbol{P}}=-i \eta(\boldsymbol{k} \hat{\boldsymbol{v}})^{s y m}-l^{2} k^{2} \varphi^{2} \hat{\boldsymbol{P}}
$$

wherein, $k^{2}=\boldsymbol{k} . \boldsymbol{k}$. and $(\boldsymbol{k} \boldsymbol{v})^{\text {sym }}$ is the symmetric traceless dyadic product of the vectors $\boldsymbol{k}$ and $\boldsymbol{v}$. 
Restricting the analysis to steady states, which is sufficient for our purpose to determine the effective viscosity in terms of the volume fraction and size of the nanoparticles, expression (20) writes as

$$
\hat{\boldsymbol{P}}\left(1+l^{2} \varphi^{2} k^{2}\right)=-i \eta(\hat{\boldsymbol{k} v})^{s y m},
$$

suggesting that the effective viscosity of the nanofluid is given by

$$
\eta_{e f f}=\eta \frac{1}{\left(1+l^{2} \varphi^{2} k^{2}\right)}
$$

with $\eta$ designating the viscosity of the nanofluid when the size effects, modelled by the corrective term in $1 /\left(1+l^{2} \varphi^{2} k^{2}\right)$ are ignored. At relatively small concentration of the nanoparticles, it is widely admitted (e.g.[1-8]), that $\eta$ is either a linear or a quadratic function of the volume fraction $\varphi$ of dispersed particles. As our main objective is to focus on the study of size effect, we shall, as a first approximation, take for $\eta$ the simplest $\varphi$-dependence, namely the linear law

$$
\eta=\eta_{f}(1+\alpha \varphi)
$$

wherein $\eta_{f}$ is the viscosity of the host fluid and $\alpha$ the enhancement coefficient, in Einstein's classical theory of suspensions [11], this coefficient is a constant given by $\alpha=2.5$. This is the value selected in the forthcoming of the present work. It is important to realize that a phenomenological description as Extended Thermodynamics cannot be used to determine the complete dependence of the transport coefficients with respect to the particles density, or temperature. This is in particular true for the viscosity $\eta$ versus particle volume-fraction density, which must resort either from experience, or from another theory, say like here, Einstein's one. 
An important feature that distinguishes the viscosity of nanofluids from classical particles dispersion in fluids is the effect caused by the presence of an amorphous overlayer encapsulating the nanoparticles [22-25, 32, 36-39]. As a consequence of liquid layering, the radius $r$ of the particles will be modified from $r$ to $r+h$ and the volume fraction of the particles from $\varphi$ to an effective volume fraction $\varphi(1+h / r)^{3}$ with $h$ standing for the thickness of the surrounding layer.

Selecting the wave number $k$ in expression (22) is a delicate task. Indeed, $k$ will be related to a characteristic length that takes into account the presence of nanoparticles. Here we find it rather naturel to take $r+h$ as reference length. A similar attitude is followed in works about the effect of nanoparticles' dispersion on the heat conductivity coefficient of nanofluids and nanocomposites [31,39-41]. Accordingly, the wave number $k$ will therefore be given by

$$
k=\frac{2 \pi}{r+h} .
$$

To summarize, the final expression of the effective viscosity of the nanofluid will take the following form obtained by combining (22), (23) and (24),

$$
\eta_{e f f}=\eta_{f} \frac{1+2.5 \varphi(1+h / r)^{3}}{1+4 \pi^{2} \varphi^{2}(1+h / r)^{4}\left(l^{2} / r^{2}\right)}
$$

for $\varphi=0, \eta_{\text {eff }}$ reduces to $\eta_{f}$ as expected. In the next section, we apply the result (25) to the problem of dispersion of lithium nanoparticles in liquid argon and alumina nanoparticles in water, respectively.

\section{Applications}




\subsection{Li nanoparticles dispersed in liquid Ar}

This example has been chosen in order to compare with a recent analysis by Rudyak and Krasnotlutskii [42] and to provide an answer to the controversial question of increase or decrease of nanofluid viscosity with the particle size. The nanoparticles are assumed to be rigid spheres uniformly dispersed in the fluid carrier, the temperature is fixed at $T=300 \mathrm{~K}$ and volume fraction of particles is small, ranging from 0.01 to 0.10 . As our aim is not to find the best dependence of the viscosity with particle concentration, we have selected for it the simplest linear law with the coefficient $\alpha=2.5$ as suggested by Einstein [11] rather than more complicated laws with ad hoc values $[1,38]$ The radius $r$ of the nanoparticles $\mathrm{Li}$ is ranging from 1 to $4 \mathrm{~nm}$. As for the mean free path $l$, we start from Matthiessen's rule stating that $1 / l=$ $1 / l_{A r-A r}+1 / l_{L i-L i}+1 / l_{A r-L}$, with $l_{A r-A r}, l_{L i-L i}, l_{A r-L i}$ designating the mean free path associated to collisions between $\mathrm{Ar}-\mathrm{Ar}, \mathrm{Li}-\mathrm{Li}$ and $\mathrm{Ar}-\mathrm{Li}$ molecules respectively. Referring to the kinetic theory [43], the mean free path is inversely proportional to the number of particles per unit volume, it follows that the main contribution will arise from the term $1 / l_{A r-A r}$. We have checked numerically that by including the other contributions of Matthiessen's law, the modifications are minute without influence on the final results, we select therefore as a first approximation $l=l_{A r-A r}$. The mean free path is found to be given by $l_{A r-A r}=(\sqrt{3 / 2}-1) r_{A r} \approx$ $0.043 \mathrm{~nm}$, after taking for $r_{A r}$ the Van der Waals radius, $r_{A r}=0.19 \mathrm{~nm}$. The value of $l_{A r-A r}$ takes into account the radius of an interstitial sphere that would fit between closely packed spheres of Ar without distorting the structure explicitly: this particular value has been selected because of the strong packing of the Ar molecules considered in [42] to which our results will be compared. Since no value for the thickness $h$ of the boundary layer surrounding $L i$ particles in Argon is found in the literature, we have assumed that $h=0.2 \mathrm{~nm}$, which is of the order of 
magnitude of the Van der Waals radius of Ar, which, by the way, lies within the range of values proposed for other fluids [44-49].

The dependence of the relative viscosity $\eta_{\text {eff }} / \eta_{f}$ on the volume fraction of Li particles is shown in Fig.1, assuming that the radius of Li nanoparticles is $r=1 \mathrm{~nm}$, the thickness of the bounding layer $h=0.2 \mathrm{~nm}$ and the mean free path in liquid Ar is $l=0.043 \mathrm{~nm}$. The results are compared with experimental data and with Einstein's model [11], which accounts only for the volume fraction of dispersed particles.

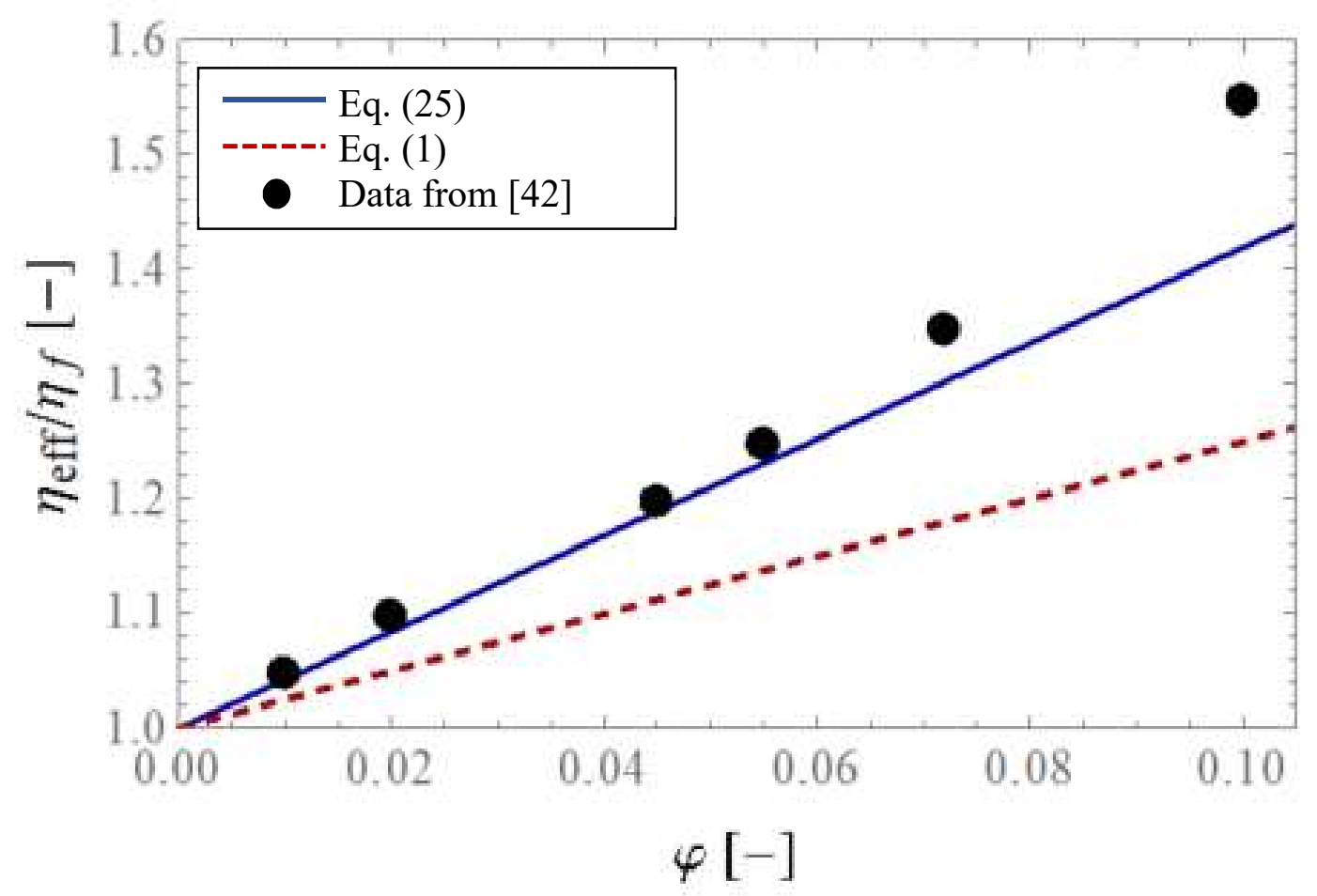

Fig. 1: Relative effective viscosity as a function of the volume fraction of Li nanoparticles with $r=1 \mathrm{~nm}$ in $300 \mathrm{~K}$ liquid Argon, comparing our model (Eq. (25)) with Einstein's relation (Eq. (1), dotted line) and data from [42] (full circles)

Numerical simulations indicate a drastic increase of effective viscosity with the volume fraction of nanoparticles in full agreement with the theoretical models developed earlier. This 
result may be interpreted by the fact that by increasing the volume fraction, one increases the interfacial surface between particles and fluid giving rise to a larger resistance to deformation, whence a higher viscosity. Our results are also shown to be in good accord with these obtained by Rudyak and Krasnolutskii [42] using a molecular dynamics method. It is seen that for volume fractions higher than 0.06 , the agreement between the model and the data from [42] is less satisfactory, which can be understood by recalling that Einstein's model is only valid for small volume fractions.

Our main objective remains the study of the behavior of viscosity as a function of the particle size. In that respect, we have analyzed the dependence of the viscosity versus the size of the Li particles for two different values of the volume fraction: $\varphi=0.02$ and 0.04 . It is seen in Fig. 2 that viscosity is decreasing with increasing dimensions, in accord with Rudyak and Krasnolutskii [42].This is a confirmation that, at least for the couple Li-Ar, a reduction of viscosity is expected with increasing particle size. This may be explained because by augmenting the dimensions of the particles, one decreases the interface between particles and host fluid whence a weaker resistance to deformation and as a consequence, reduction of viscosity. It is worth mentioning that the dependence of viscosity with particles' dimensions is weak as soon as the diameter exceeds $4 \mathrm{~nm}$, meaning that for large particles, viscosity is independent of the size of dispersed particles, as confirmed by the next example. 


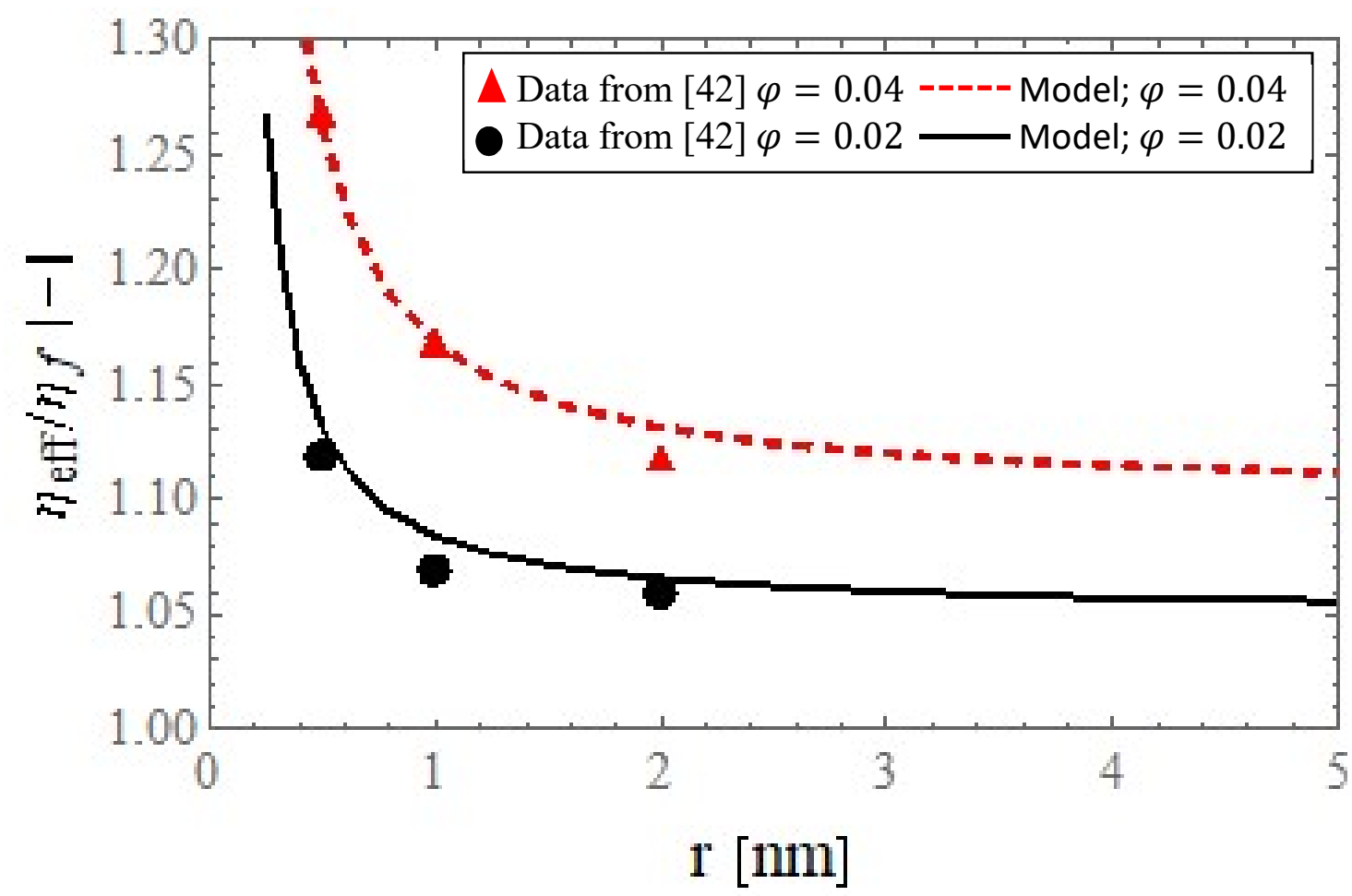

Fig. 2: Relative effective viscosity as a function of the radius $r$ of Li nanoparticles in $300 \mathrm{~K}$ liquid Argon, comparing our model (Eq. (25)) with data from [42] for two volume fractions: $\varphi=0.02$ (black solid line) and $\varphi=0.04$ (red dotted line). Full circles and triangles represent the data from [42].

\subsection{Alumina $\mathrm{Al}_{2} \mathrm{O}_{3}$ particles in water}

For alumina nanoparticles dispersed in water, it is found [49] that the thickness of the boundary layer around $\mathrm{Al}_{2} \mathrm{O}_{3}$ particles is roughly $h=4 \mathrm{~nm}$. The mean free path $l$ is taken to be that of the average distance between water molecules [50], i.e. $l=0.31 \mathrm{~nm}$. In Fig. 3, the theoretical effective viscosity of the nanofluid is compared to experimental values [51] for two different radii of $\mathrm{Al}_{2} \mathrm{O}_{3}$ particles: $\mathrm{r}=4$ and $21.5 \mathrm{~nm}$. For the sake of completeness, we have also drawn the results provided by Einstein's theory. It is still shown that viscosity is increasing with increasing volume fraction and decreases with larger size. The latter is confirmed by the results 
reported in Fig. 4, where we have represented the dependence of the relative effective viscosity on the particle radius. It is also worth noting that our model fits satisfactorily the few experimental data available in the literature, the less good results for the largest volume fraction may be explained by the use of Einstein's model.

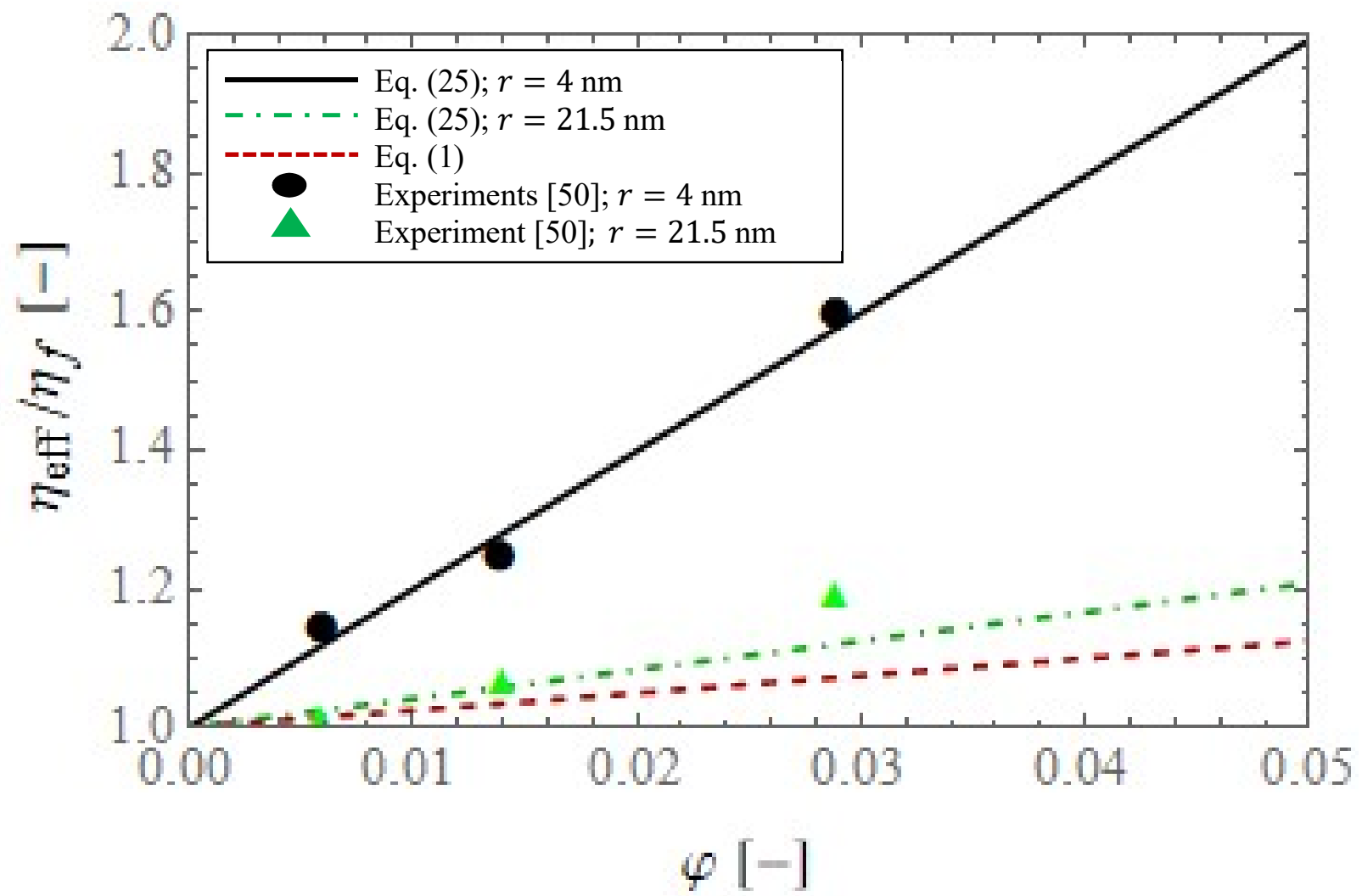

Fig. 3: Relative viscosity as a function of the volume fraction of $\mathrm{Al}_{2} \mathrm{O}_{3}$ nanoparticles in water at ambient conditions, comparing our model (Eq. (25)) with Eq. (1) and experimental values [50] for two nanoparticles radii: $\mathrm{r}=4 \mathrm{~nm}$ and $21.5 \mathrm{~nm}$. 


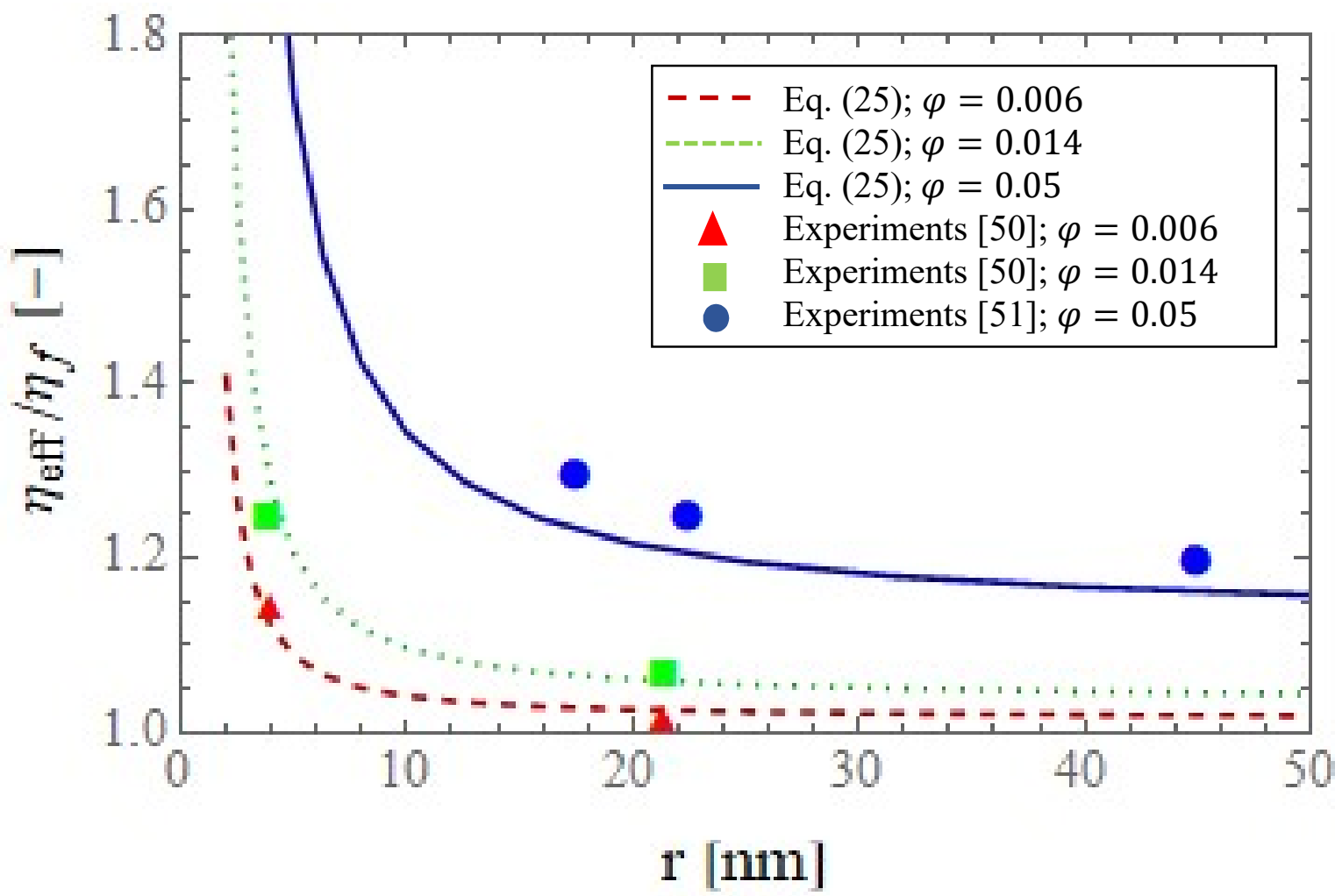

Fig. 4: Relative effective viscosity as a function of the radius of $\mathrm{Al}_{2} \mathrm{O}_{3}$ nanoparticles in water at ambient conditions, comparing our model (Eq. (25)) with experimental values [50,51] for three different volume fractions of $\varphi=0.006,0.014$ and 0.05 .

\section{Summary and final comments.}

Our main purpose is to propose a new analytical expression for the effective viscosity of dilute hard sphere nanoparticle suspensions by using a theoretical thermodynamically-based model. In particular, it was also our aim to shed some light on the controversy between people who assert that viscosity decreases with the dimensions of the nanoparticles and their detractors who claim the opposite. Our approach confirms the first option. This was achieved by appealing to an original model, wherein use was made of the following ingredients: 
- Einstein's model for the dependence of the viscosity with respect to the volume fraction of solid particles is taken for granted.

- Use of higher order moments of the stress tensor in the framework of EIT to account for the smallness of particles.

- The presence of a hydrodynamic layer around the nanoparticles is assumed.

- Coupling of all three previous effects: volume fraction, particle's size and liquid layering.

The main result of our analysis is embedded in relation (25). Accordingly, it is found that the effective viscosity of nanofluids increases with the volume fraction of nanoparticles and decreases with particle's size. It is interesting to notice that an opposite behavior was observed in the case of the thermal conductivity of nanocomposites which decreases with volume fraction but increases with increasing size of nanoparticles (e.g. [31]). Our thermodynamics modelling of the size effect on the viscosity in the mixture Li-Ar is in agreement with the results obtained by Ruydak and Krassnolutskii [42] based on molecular dynamics simulation and experiments. Our approach is also in accord with the data reported for the more classical nanofluids, involving alumina particles dispersed in water $[48,51]$.

It should be kept in mind that the present approach is based on a phenomenological thermodynamic formalism referred to as Extended Irreversible Thermodynamics (EIT). Like any other macroscopic theory, EIT is not sufficient per se to provide a complete relationship between viscosity and the volume fraction of nanoparticles and/or to account for the formation of an interfacial layer at the fluid-particle interface. These data have to be borrowed from other formalisms, like Einstein's theory, molecular dynamics simulations, statistical physics, kinetic theory or experiences. Here, our first objective was to answer the question of the role of 
particles' size on the nanofluid viscosity, we have seen that in that respect, EIT is able to provide a sound estimate.

In the future, it is expected to check to which extent the results are modified by using more elaborate models than Einstein's one to describe the nanoparticles density dependence. In that perspective, it would be interesting to examine the influence of approaches like these of Krieger and Dougherty [9] or Batchelor [12] among others.

\section{Acknowledgements}

BelSPo is acknowledged for financial support via the Prodex programme.

\section{References}

[1] V.Y. Rudyak, Viscosity of nanofluids-Why it is not described by the classical theories, Adv. in Nanoparticles, 2 (2013), 266-279. doi.org/10.4236/anp.2013.23037

[2] N.V. Shanker, M. C. Shekar Reddy, V.V Basavo Rao, On prediction of viscosity of nanofluids for low volume fractions of nanoparticles, Int. .J. Eng. Res. and Technology 1(8) (2012) 1-10.

[3] M. Masomi, N. Sohrabi, A. Behzadmehr, A new model for calculating the effective viscosity of nanofluids, J. Appl. Phys. D 42 (2009) 055501.

[4] P.C. Mishra, S. Mukherjee, S.K. Nayak, A. Panda, A brief review on viscosity of nanofluids, Int. Nano Lett. 4 (2014) 109-120.

[5] H. Chen, Y. Ding and C. Tan, Rheological behavior of nanofluids, New Journal of Physics 9 (2007) 367/24 pp.

[6] P. Prasher, D. Song and J. Wang, Measurements of nanofluid viscosity and its implications for thermal applications, Appl. Phys. Lett. 89 (2006) ID: 133108. doi:10.1063/1.2356113.

[7] A. Graham, On the viscosity of suspensions of solid spheres, Appl. Sci. Res.37 (1981). 275-286. doi:10.1007/BF00951252. 
[8] I.M. Mahbubul, R. Saidur, M.A. Amalina, Latest developments on the viscosity of nanofluids, Int. J. Heat Mass Transfer 55(4) (2012) 874-885.

[9] J.M. Krieger, T.J. Dougherty, A mechanism for non-Newtonian flow in suspensions of rigid spheres. Transactions Soc. Rheol. 3(1) (1957) 137-152.

[10] J. Szymański, A. Wilk, R. Hołyst, G. Roberts, K. Sinclair, A. Kowalski, Micro- and macro-shear viscosity in dispersed lamellar phases, J. Non-Newton. Fluid Mech. 148 (2008) 134-140.

[11] A. Einstein, Eine neue bestimmung der molekul-dimension, Annal. Phys. 34(2),(1906) 289-306.

[12] G.K. Batchelor, Effect of Brownian-motion on the bulk stress in a suspension of spherical particles, J. Fluid Mech. 83 (1977) 97-117.

[13] Y. He, Y. Jin, H. Chen, Y. Ding, D. Cang and H. Lu, Heat transfer and flow behaviour of aqueous suspensions of $\mathrm{TiO} 2$ nanoparticles (nanofluids) flowing upward through a vertical pipe, Int. J. Heat and Mass Transfer, 50 (2007) 2272-2281. doi:10.1016/j.ijheatmasstransfer.2006.10.024.

[ 14 ] C. T. Nguyen, F. Desgranges, G. Roy, N. Galanis, T. Marer, S. Boucher and H. Mintsa, Temperature and particle-size dependent viscosity data for water-based nanofluids - hysteresis phenomenon, Int. J. Heat and Fluid Flow, 28 (2007) 1492-1506. doi:10.1016/j. ijheatfluidflow.2007.02.004.

[15]] P. K. Namburu, D. P. Kulkarni, A. Dandekar and D. K. Das, Experimental investigation of viscosity and specific heat and Silicon Dioxide nanofluids," Micro \& Nano Letters, 2 (3) (2007) 67-71.

[16] J. Chevalier, O. Tillement and F. Ayela, Rheological properties of nanofluids flowing through microchannels, Appl. Phys. Lett. 91(23) (2008) Article ID: 233103.

[17] E. V. Timofeeva, D. S. Smith, W. Yu, D. M. France, D. Singh and J. L. Routbo, "Particle size and interfacial effects on thermo-physical and heat transfer characteristics of water-based a-SiC nanofluids, Nanotechnology 21(21)2010, Article ID: 215703.

[18] V. Y. Rudyak, A. A. Belkin and V. V. Egorov, On the effective viscosity of nanosuspensions," Techn. Phys.54 (8) (2009) 1102-1109. .

[19] D Jou, J. Casas-Vàzquez, G. Lebon, Extended Irreversible Thermodynamics. 4th edition. Springer, New York, 2010. 
[20] G. Lebon, D. Jou, J. Casas-Vazquez, Understanding Non-Equilibrium Thermodynamics, Springer, Berlin, 2008.

[21] G. Lebon, D. Jou, Early history of extended irreversible thermodynamics (1953-1983): An exploration beyond local equilibrium and classical transport theory, Eur. Phys. Journal H 5 (2014) DOI:10.114/epjh/e2014-500033-0 (36 pp)

[22] S.U.S. Choi, Enhancing thermal conductivity of fluids with nanoparticles, In: D. A. Siginer and H. P. Wang, Eds., Developments Applications of Non-Newtonian Flows, Vol. 231, ASME, New York 66 (1995).99-105.

[23] W. Yu, S.U.S Choi, The role of interfacial layers in the enhanced thermal conductivity of nanofluids: A renovated Maxwell model. J. Nanopart. Res. 5 (2003) 167-171.

[24] H. Xie, M. Fujii, X. Zhang, Effect of interfacial nanolayer on the effective thermal conductivity of nanoparticle-fluid mixture. Int. J. Heat Mass Transf. 48 (2005) 2926-2932.

[25] I. Prigogine, Introduction to Thermodynamics of Irreversible Processes, Interscience, New York, 1961.

[26] M. Boukary, G. Lebon, A comparative analysis of binary fluid mixtures by extended thermodynamics and the kinetic theory, Physica 137A (1986) 546-572.

[27] D. Jou, J. Casas-Vazquez, M. Criado-Sancho, Thermodynamics of Fluids under Flow, 2nd edition, Springer, Dordrecht, Heidelberg, London, New York, 2011.

[28] N. Depireux, G. Lebon, An extended thermodynamic modelling of non-Fickian diffusion, J. Non-Newt. Fluid Mech. 96 (2001) 105-117.

[29]. G. Lebon, T. Desaive, P.C. Dauby, A unified extended thermodynamic description of diffusion, thermodiffusion, suspensions, and porous media, J. Applied Mech.73 (2006) 16-20.

[30] D. Jou, J. Casas-Vazquez, G. Lebon, M. Grmela, A phenomenological scaling approach for heat transport in nano-systems, Appl. Math. Lett. 18 (2005) 963-967.

[31] G. Lebon, H. Machrafi, M.Grmela, An extended irreversible thermodynamic modelling of size-dependent thermal conductivity of spherical nanoparticles dispersed in homogeneous media. Proc. R. Soc. A 471 (2015) 20150144.

[32] H. Machrafi, G. Lebon, The role of several heat transfer mechanisms on the enhancement of thermal conductivity in nanofluids, Continuum Mech. Thermodyn. (2016) DOI $10.1007 / \mathrm{s} 00161-015-0488-4$. 
[33] H. Machrafi, G. Lebon, General constitutive equations of heat transport at small length and high frequencies with extension to mass and electrical scales transport, Appl. Math. Lett. 22 (2016) 30-37.

[34] N. Clarke, T.C.B. McLeish, Shear flow on phase separation of entangled polymer blends, Phys. Rev. 57 (1998) R3731-R3734.

[35] R.A. Guyer, J.A. Krumhansl, Solution of the linearized Boltzmann phonon equation, Phys. Rev. 148 (1966) 766-778.

[36] R. Pasrija, S.Srivastava, The interfacial layer effect on thermal conductivity of nanocolloidal dispersions. Int. J. Appl. Phys. Math. 4 (2014) 1-4.

[37] M. Kole, T.K.Dey, Role of interfacial layer and clustering on the effective thermal conductivity of CuO-gear oil nanofluids, Exp. Thermal Fluid Sci. 35 (2011) 1490-1495.

[38] E.E. Michaelidis, Transport properties of nanofluids. A critical review, J. Non-Equilib. Thermodyn. 38 (2013) 1-31.

[39] F.X. Alvarez, D. Jou, Memory and the non-local effects in heat transport from diffusive and ballistic regimes, Appl. Phys. Lett. 90 (2007) 083109

[40] A. Sellitto, V.A. Cimmelli, D. Jou, Mesoscopic theories of heat transport in nanosystems, Springer, Berlin, 2016.

[41] G. Lebon, H. Machrafi, Effective thermal conductivity of nanostuctures : a review, Atti Accademia Peloritana dei Perilocanti, to appear 2018.

[42] V.Y. Rudyak, S.L.Krasnolutskii, Dependence of the viscosity of nanofluids on nanoparticle size and material, Phys. Lett. A378 (2014)1845-1849.

[43] S. Chapman, T.C. Cowling, The mathematical theory of non-uniform gases, Cambridge Univ. Press, Cambridge, 1970.

[44] T. Hashimoto, M. Fujimura, H Kawai, Domain-boundary structure of styrene-isoprene block co-polymer films cast from solutions. Macromolecules 13 (1980) 660-669.

[45] Z.H. Li, Y.J. Gong, M. Pu, D. Wu, Y.H. Sun, J.Wang, Y.Liu, B.Z.Dong, Determination of interfacial layer thickness of a pseudo two-phase system by extension of the Debye equation. J. Phys. D: Appl. Phys. 34 (2001)2085-2088.

[46] C.J. Yu, A.G. Richter, A. Datta, M.K. Durbin, P. Dutta, Molecular layering in a liquid on a solid substrate: an X-ray reflectivity study. Phys. B 283, (2000)27-31. 
[47] L Xue, P. Keblinski, S.R Phillipot, S.U.S Choi, J.A. Eastman, Effect of liquid layering at the liquid-solid interface on thermal transport. Int. J. Heat Mass Transf. 47 (2004) 4277-4284.

[48] E. Firlar, S. Cinar, S. Kashyap, M. Akinc, T. Prozorov, Direct visualization of the hydration layer on alumina nanoparticles with the fluid cell STEM in situ, Sci .Reports 5 (2015) 9830-

[49] S.J. Perkins, Protein volumes and hydration effects. The calculations of partial specific volumes, neutron scattering match points and 280-nm absorption coefficients for proteins and glycoproteins from amino acid sequences, Eur. J. Biochem. 157 (1986), 169-180.

[50] M.J. Pastoriza-Gallego, C. Casanova, R. Páramo, B. Barbés, J.L. Legido, M.M. Piñeiro, A study on stability and thermophysical properties (density and viscosity) of $\mathrm{Al}_{2} \mathrm{O}_{3}$ in water nanofluid, J. Appl. Phys. 106 (2009) 064301.

[51] W.Q. Lu, Q.M. Fan, Study for the particle's scale effect on some thermophysical properties of nanofluids by a simplified molecular dynamics method, Engineering Analysis with Boundary Elements 32 (2008) 282-289. 